\title{
NEW OSTROWSKI TYPE INEQUALITY FOR TRIPLE INTEGRALS
}

\section{\|\|$\|$}

\author{
B. G. PACHPATTE
}

\begin{abstract}
The main aim of this paper is to establish a new Ostrowski type inequality for triple integrals by using a fairly elementary analysis. The discrete version of the result is also given.
\end{abstract}

\section{Introduction}

The well known Ostrowski's inequality [7] can be stated as follows (see slso [6, p.469]).

Let $f:[a, b] \rightarrow R$ be continuous on $[a, b]$ and differentiable on $(a, b)$ whose derivative $f^{\prime}:(a, b) \rightarrow R$ is bounded on $(a, b)$ i.e., $\left\|f^{\prime}\right\|_{\infty}=\sup _{t \in(a, b)}\left|f^{\prime}(t)\right|<\infty$, then

$$
\left|f(x)-\frac{1}{b-a} \int_{a}^{b} f(t) d t\right| \leq\left[\frac{1}{4}+\frac{\left(x-\frac{a+b}{2}\right)^{2}}{(b-a)^{2}}\right](b-a)\left\|f^{\prime}\right\|_{\infty},
$$

for all $x \in[a, b]$.

In 2000, Pachpatte [8] obtained the following Ostrowski type inequality for triple integrals.

Let $\Delta=[a, k] \times[b, m] \times[c, n]$ for $a, b, c, k, m, n$ in $R_{+}$and $f(r, s, t)$ be differentiable on $\Delta$. Denote the partial derivatives by $D_{1} f(r, s, t)=\frac{\partial}{\partial r} f(r, s, t), D_{2} f(r, s, t)=$ $\frac{\partial}{\partial s} f(r, s, t), D_{3} f(r, s, t)=\frac{\partial}{\partial t} f(r, s, t)$ and $D_{3} D_{2} D_{1} f(r, s, t)=\frac{\partial^{3}}{\partial t \partial s \partial r} f(r, s, t)$. Let $F(\Delta)$ be the class of continuous functions $f: \Delta \rightarrow R$ for which $D_{1} f, D_{2} f, D_{3} f, D_{3} D_{2} D_{1} f$ exist and are continuous on $\Delta$. For $f \in F(\Delta)$ we have

$$
\begin{aligned}
& \mid \int_{a}^{k} \int_{b}^{m} \int_{c}^{n} f(r, s, t) d t d s d r-\frac{1}{8}(k-a)(m-b)(n-c)[f(a, b, c)+f(k, m, n)] \\
& \quad+\frac{1}{4}(m-b)(n-c) \int_{a}^{k}[f(r, b, c)+f(r, m, n)+f(r, m, c)+f(r, b, n)] d r \\
& \quad+\frac{1}{4}(k-a)(n-c) \int_{b}^{m}[f(a, s, c)+f(k, s, n)+f(a, s, n)+f(k, s, c)] d s
\end{aligned}
$$

Received November 22, 2005.

2000 Mathematics Subject Classification. 26D15, 26D20.

Key words and phrases. Ostrowski type inequality, triple integrals, discrete version, partial derivatives, difference operators, identity. 


$$
\begin{aligned}
& +\frac{1}{4}(k-a)(m-b) \int_{c}^{n}[f(a, b, t)+f(k, m, t)+f(k, b, t)+f(a, m, t)] d t \\
& -\frac{1}{2}(k-a) \int_{b}^{m} \int_{c}^{n}[f(a, s, t)+f(k, s, t)] d t d s \\
& -\frac{1}{2}(m-b) \int_{a}^{k} \int_{c}^{n}[f(r, b, t)+f(r, m, t)] d t d r \\
& -\frac{1}{2}(n-c) \int_{a}^{k} \int_{b}^{m}[f(r, s, c)+f(r, s, n)] d s d r \mid \\
& \leq \frac{1}{8}(k-a)(m-b)(n-c) \int_{a}^{k} \int_{b}^{m} \int_{c}^{n}\left|D_{3} D_{2} D_{1} f(r, s, t)\right| d t d s d r .
\end{aligned}
$$

In [8], the inequality (1.2) and its discrete version are established by using elementary analysis. In [13] Sofo has given a refinement of the inequality (1.2) by using Peano kernels. For Ostrowski type inequalities in several independent variables, we refer the interested readers to $[1-5,9-12]$. The main purpose of the present paper is to establish a new Ostrowski type inequality involving functions of three independent variables and their partial derivatives. The discrete version of the main result is also given.

\section{Statement of Results}

In what follows $R$ denotes the set of real numbers. We use the notation $H=$ $\left[a_{1}, b_{1}\right] \times\left[a_{2}, b_{2}\right] \times\left[a_{3}, b_{3}\right]\left(a_{1}<b_{1}, a_{2}<b_{2}, a_{3}<b_{3}\right)$ for $a_{1}, a_{2}, a_{3}, b_{1}, b_{2}, b_{3}$ in $R$. If $h=$ $h(r, s, t)$ is a differentiable function defined on $H$, then its partial derivatives are denoted by $D_{1} h=\frac{\partial}{\partial r} h, D_{2} h=\frac{\partial}{\partial s} h, D_{3} h=\frac{\partial}{\partial t} h, D_{1} D_{2} h=\frac{\partial^{2}}{\partial r \partial s} h, D_{2} D_{3} h=\frac{\partial^{2}}{\partial s \partial t} h$, $D_{3} D_{1} h=\frac{\partial^{2}}{\partial t \partial r} h$, and $D_{3} D_{2} D_{1} h=\frac{\partial^{3}}{\partial t \partial s \partial r} h$. We denote by $F(H)$ the class of continuous functions $h: H \rightarrow R$ for which $D_{1} h, D_{2} h, D_{3} h, D_{1} D_{2} h, D_{2} D_{3} h, D_{3} D_{1} h$, $D_{3} D_{2} D_{1} h$ exist and are continuous on $H$. Let $N$ denote the set of natural numbers, $A=\{1,2, \ldots, a+1\}, B=\{1,2, \ldots, b+1\}, C=\{1,2, \ldots, c+1\}$ for $a, b, c$ in $N$ and $E=A \times B \times C$. For a function $h=h(x, y, z): N^{3} \rightarrow R$ we define the difference operators $\Delta_{1} h=h(x+1, y, z)-h(x, y, z), \Delta_{2} h=h(x, y+1, z)-h(x, y, z)$, $\Delta_{3} h=h(x, y, z+1)-h(x, y, z), \Delta_{1} \Delta_{2} h=\Delta_{1}\left(\Delta_{2} h\right), \Delta_{2} \Delta_{3} h=\Delta_{2}\left(\Delta_{3} h\right), \Delta_{3} \Delta_{1} h=$ $\Delta_{3}\left(\Delta_{1} h\right), \Delta_{3} \Delta_{2} \Delta_{1} h=\Delta_{3}\left(\Delta_{2} \Delta_{1} h\right)$. We denote by $G(E)$ the class of functions $h=$ $h(x, y, z): E \rightarrow R$ for which $\Delta_{1} h, \Delta_{2} h, \Delta_{3} h, \Delta_{1} \Delta_{2} h, \Delta_{2} \Delta_{3} h, \Delta_{3} \Delta_{1} h, \Delta_{3} \Delta_{2} \Delta_{1} h$ exist on $E$. We assume that $h(x, y, z)=0$ for $(x, y, z) \notin E$ and also use the usual convention that, empty sum is taken to be 0 .

Our main result is given in the following theorem.

Theorem 1. Let $f \in F(H)$. Then

$$
\mid f(x, y, z)-\left[\frac{1}{b_{1}-a_{1}} \int_{a_{1}}^{b_{1}} f(r, y, z) d r+\frac{1}{b_{2}-a_{2}} \int_{a_{2}}^{b_{2}} f(x, s, z) d s\right.
$$




$$
\begin{aligned}
& \left.+\frac{1}{b_{3}-a_{3}} \int_{a_{3}}^{b_{3}} f(x, y, t) d t\right] \\
& +\left[\frac{1}{\left(b_{1}-a_{1}\right)\left(b_{2}-a_{2}\right)} \int_{a_{1}}^{b_{1}} \int_{a_{2}}^{b_{2}} f(r, s, z) d s d r\right. \\
& +\frac{1}{\left(b_{1}-a_{1}\right)\left(b_{3}-a_{3}\right)} \int_{a_{1}}^{b_{1}} \int_{a_{3}}^{b_{3}} f(r, y, t) d t d r \\
& \left.+\frac{1}{\left(b_{2}-a_{2}\right)\left(b_{3}-a_{3}\right)} \int_{a_{2}}^{b_{2}} \int_{a_{3}}^{b_{3}} f(x, s, t) d t d s\right] \\
& -\frac{1}{\left(b_{1}-a_{1}\right)\left(b_{2}-a_{2}\right)\left(b_{3}-a_{3}\right)} \int_{a_{1}}^{b_{1}} \int_{a_{2}}^{b_{2}} \int_{a_{3}}^{b_{3}} f(r, s, t) d t d s d r \mid \\
& \leq \frac{1}{\left(b_{1}-a_{1}\right)\left(b_{2}-a_{2}\right)\left(b_{3}-a_{3}\right)} \\
& \times \int_{a_{1}}^{b_{1}} \int_{a_{2}}^{b_{2}} \int_{a_{3}}^{b_{3}}\left|\int_{r}^{x} \int_{s}^{y} \int_{t}^{z} D_{3} D_{2} D_{1} f(u, v, w) d w d v d u\right| d t d s d r
\end{aligned}
$$

for all $(x, y, z) \in H$.

The following Corollary holds.

Corollary. Let $f$ be as in Theorem 1. Then

$$
\begin{aligned}
\mid f & \left(\frac{a_{1}+b_{1}}{2}, \frac{a_{2}+b_{2}}{2}, \frac{a_{3}+b_{3}}{2}\right)-\left[\frac{1}{b_{1}-a_{1}} \int_{a_{1}}^{b_{1}} f\left(r, \frac{a_{2}+b_{2}}{2}, \frac{a_{3}+b_{3}}{2}\right) d r\right. \\
& \left.+\frac{1}{b_{2}-a_{2}} \int_{a_{2}}^{b_{2}} f\left(\frac{a_{1}+b_{1}}{2}, s, \frac{a_{3}+b_{3}}{2}\right) d s+\frac{1}{b_{3}-a_{3}} \int_{a_{3}}^{b_{3}} f\left(\frac{a_{1}+b_{1}}{2}, \frac{a_{2}+b_{2}}{2}, t\right) d t\right] \\
& +\left[\frac{1}{\left(b_{1}-a_{1}\right)\left(b_{2}-a_{2}\right)} \int_{a_{1}}^{b_{1}} \int_{a_{2}}^{b_{2}} f\left(r, s, \frac{a_{3}+b_{3}}{2}\right) d s d r\right. \\
& +\frac{1}{\left(b_{1}-a_{1}\right)\left(b_{3}-a_{3}\right)} \int_{a_{1}}^{b_{1}} \int_{a_{3}}^{b_{3}} f\left(r, \frac{a_{2}+b_{2}}{2}, t\right) d t d r \\
& \left.+\frac{1}{\left(b_{2}-a_{2}\right)\left(b_{3}-a_{3}\right)} \int_{a_{2}}^{b_{2}} \int_{a_{3}}^{b_{3}} f\left(\frac{a_{1}+b_{1}}{2}, s, t\right) d t d s\right] \\
& -\frac{1}{\left(b_{1}-a_{1}\right)\left(b_{2}-a_{2}\right)\left(b_{3}-a_{3}\right)} \int_{a_{1}}^{b_{1}} \int_{a_{2}}^{b_{2}} \int_{a_{3}}^{b_{3}} f(r, s, t) d t d s d r \mid \\
\leq & \frac{\left(b_{1}-a_{1}\right)\left(b_{2}-a_{2}\right)\left(b_{3}-a_{3}\right)}{64}\left\|D_{3} D_{2} D_{1} f\right\|_{\infty},
\end{aligned}
$$

where

$$
\left\|D_{3} D_{2} D_{1} f\right\|_{\infty}=\sup _{(u, v, w) \in H}\left|D_{3} D_{2} D_{1} f(u, v, w)\right|<\infty .
$$

By taking $x=\frac{a_{1}+b_{1}}{2}, y=\frac{a_{2}+b_{2}}{2}, z=\frac{a_{3}+b_{3}}{2}$ in (2.1) and simple computation, we get the desired inequality in (2.2). 
The discrete version of Theorem 1 is embodied in the following theorem.

Theorem 2. Let $f \in G(E)$. Then

$$
\begin{aligned}
\mid f(k, m, n)-\left[\frac{1}{a} \sum_{r=1}^{a} f(r, m, n)+\frac{1}{b} \sum_{s=1}^{b} f(k, s, n)+\frac{1}{c} \sum_{t=1}^{c} f(k, m, t)\right] \\
+\left[\frac{1}{a b} \sum_{r=1}^{a} \sum_{s=1}^{b} f(r, s, n)+\frac{1}{a c} \sum_{r=1}^{a} \sum_{t=1}^{c} f(r, m, t)+\frac{1}{b c} \sum_{s=1}^{b} \sum_{t=1}^{c} f(k, s, t)\right] \\
\quad-\frac{1}{a b c} \sum_{r=1}^{a} \sum_{s=1}^{b} \sum_{t=1}^{c} f(r, s, t) \mid \\
\leq \frac{1}{a b c} \sum_{r=1}^{a} \sum_{s=1}^{b} \sum_{t=1}^{c}\left|\sum_{u=r}^{k-1} \sum_{v=s}^{m-1} \sum_{w=t}^{n-1} \Delta_{3} \Delta_{2} \Delta_{1} f(u, v, w)\right|
\end{aligned}
$$

for all $(k, m, n) \in E$.

\section{Proof of Theorem 1}

The proof is based on the following identity

$$
\begin{aligned}
I= & f(x, y, z)-[f(r, y, z)+f(x, s, z)+f(x, y, t)] \\
& +[f(r, s, z)+f(r, y, t)+f(x, s, t)]-f(r, s, t),
\end{aligned}
$$

for $(x, y, z),(r, s, t) \in H$, where

$$
I=\int_{r}^{x} \int_{s}^{y} \int_{t}^{z} D_{3} D_{2} D_{1} f(u, v, w) d w d v d u .
$$

From (3.2) it is easy to observe that

$$
\begin{aligned}
I & =\int_{r}^{x} \int_{s}^{y} D_{2} D_{1} f(u, v, z) d v d u-\int_{r}^{x} \int_{s}^{y} D_{2} D_{1} f(u, v, t) d v d u \\
& =I_{1}-I_{2} .
\end{aligned}
$$

By simple computation we have

$$
\begin{aligned}
I_{1} & =\int_{r}^{x} \int_{s}^{y} D_{2} D_{1} f(u, v, z) d v d u \\
& =\int_{r}^{x} D_{1} f(u, y, z) d u-\int_{r}^{x} D_{1} f(u, s, z) d u \\
& =f(x, y, z)-f(r, y, z)-f(x, s, z)+f(r, s, z) .
\end{aligned}
$$

Similarly, we have

$$
I_{2}=f(x, y, t)-f(r, y, t)-f(x, s, t)+f(r, s, t) .
$$


Using (3.4) and (3.5) in (3.3) we get (3.1).

Integrating both sides of $(3.1)$ with respect to $(r, s, t)$ over $H$ and rewriting we get

$$
\begin{aligned}
& f(x, y, z)-\left[\frac{1}{b_{1}-a_{1}} \int_{a_{1}}^{b_{1}} f(r, y, z) d r+\frac{1}{b_{2}-a_{2}} \int_{a_{2}}^{b_{2}} f(x, s, z) d s\right. \\
&\left.+\frac{1}{b_{3}-a_{3}} \int_{a_{3}}^{b_{3}} f(x, y, t) d t\right] \\
&+\left[\frac{1}{\left(b_{1}-a_{1}\right)\left(b_{2}-a_{2}\right)} \int_{a_{1}}^{b_{1}} \int_{a_{2}}^{b_{2}} f(r, s, z) d s d r\right. \\
&+\frac{1}{\left(b_{1}-a_{1}\right)\left(b_{3}-a_{3}\right)} \int_{a_{1}}^{b_{1}} \int_{a_{3}}^{b_{3}} f(r, y, t) d t d r \\
&+\frac{1}{\left(b_{2}-a_{2}\right)\left(b_{3}-a_{3}\right)} \int_{a_{2}}^{b_{2}} \int_{a_{3}}^{b_{3}} f(x, s, t) d t d s \\
&\left.\quad-\frac{1}{\left(b_{1}-a_{1}\right)\left(b_{2}-a_{2}\right)\left(b_{3}-a_{3}\right)} \int_{a_{1}}^{b_{1}} \int_{a_{2}}^{b_{2}} \int_{a_{3}}^{b_{3}} f(r, s, t) d t d s d r\right] \\
&= \frac{1}{\left(b_{1}-a_{1}\right)\left(b_{2}-a_{2}\right)\left(b_{3}-a_{3}\right)} \\
& \times \int_{a_{1}}^{b_{1}} \int_{a_{2}}^{b_{2}} \int_{a_{3}}^{b_{3}}\left\{\int_{r}^{x} \int_{s}^{y} \int_{t}^{z} D_{3} D_{2} D_{1} f(u, v, w) d w d v d u\right\} d t d s d r
\end{aligned}
$$

for $(x, y, z) \in H$. From (3.6) and using the properties of modulus and integrals, we get the desired inequality in (2.1). The proof is complete.

\section{Proof of Theorem 2}

We first prove the following identity

$$
\begin{aligned}
S= & f(k, m, n)-[f(r, m, n)+f(k, s, n)+f(k, m, t)] \\
& +[f(r, s, n)+f(r, m, t)+f(k, s, t)]-f(r, s, t),
\end{aligned}
$$

for $(k, m, n),(r, s, t) \in E$, where

$$
S=\sum_{u=r}^{k-1} \sum_{v=s}^{m-1} \sum_{w=t}^{n-1} \Delta_{3} \Delta_{2} \Delta_{1} f(u, v, w) .
$$

From (4.2), by simple calculation we have

$$
\begin{aligned}
S & =\sum_{u=r}^{k-1} \sum_{v=s}^{m-1}\left[\sum_{w=t}^{n-1}\left\{\Delta_{2} \Delta_{1} f(u, v, w+1)-\Delta_{2} \Delta_{1} f(u, v, w)\right\}\right] \\
& =\sum_{u=r}^{k-1} \sum_{v=s}^{m-1} \Delta_{2} \Delta_{1} f(u, v, n)-\sum_{u=r}^{k-1} \sum_{v=s}^{m-1} \Delta_{2} \Delta_{1} f(u, v, t)
\end{aligned}
$$




$$
=S_{1}-S_{2}
$$

By simple calculation we have

$$
\begin{aligned}
S_{1} & =\sum_{u=r}^{k-1} \sum_{v=s}^{m-1} \Delta_{2} \Delta_{1} f(u, v, n) \\
& =\sum_{u=r}^{k-1}\left[\sum_{v=s}^{m-1}\left\{\Delta_{1} f(u, v+1, n)-\Delta_{1} f(u, v, n)\right\}\right] \\
& =\sum_{u=r}^{k-1} \Delta_{1} f(u, m, n)-\sum_{u=r}^{k-1} \Delta_{1} f(u, s, n) \\
& =\sum_{u=r}^{k-1}\{f(u+1, m, n)-f(u, m, n)\}-\sum_{u=r}^{k-1}\{f(u+1, s, n)-f(u, s, n)\} \\
& =f(k, m, n)-f(r, m, n)-f(k, s, n)+f(r, s, n) .
\end{aligned}
$$

Similarly, we have

$$
S_{2}=f(k, m, t)-f(r, m, t)-f(k, s, t)+f(r, s, t) .
$$

Using (4.4) and (4.5) in (4.3) we get (4.1).

Summing both sides of (4.1) first with respect to $t$ from 1 to $c$, then with respect to $s$ from 1 to $b$ and finally with respect to $r$ from 1 to $a$ and rewriting we have

$$
\begin{aligned}
f(k, m, n)- & {\left[\frac{1}{a} \sum_{r=1}^{a} f(r, m, n)+\frac{1}{b} \sum_{s=1}^{b} f(k, s, n)+\frac{1}{c} \sum_{t=1}^{c} f(k, m, t)\right] } \\
+ & {\left[\frac{1}{a b} \sum_{r=1}^{a} \sum_{s=1}^{b} f(r, s, n)+\frac{1}{a c} \sum_{r=1}^{a} \sum_{t=1}^{c} f(r, m, t)+\frac{1}{b c} \sum_{s=1}^{b} \sum_{t=1}^{c} f(k, s, t)\right] } \\
& -\frac{1}{a b c} \sum_{r=1}^{a} \sum_{s=1}^{b} \sum_{t=1}^{c} f(r, s, t) \\
= & \frac{1}{a b c} \sum_{r=1}^{a} \sum_{s=1}^{b} \sum_{t=1}^{c}\left\{\sum_{u=r}^{k-1} \sum_{v=s}^{m-1} \sum_{w=t}^{n-1} \Delta_{3} \Delta_{2} \Delta_{1} f(u, v, w)\right\}
\end{aligned}
$$

for all $(k, m, n) \in E$. From (4.6) and using the properties of modulus and sums we get the required inequality in (2.3). The proof is complete.

\section{References}

[1] G. A. Anastassiou, Multivariate Ostrowski type inequalities, Acta Math. Hungar. 76(1997), 267-278. 
[2] N. S. Barnett and S. S. Dragomir, An Ostrowski type inequality for double integrals and applications for cubature formulae, Soochow J. Math. 27(2001), 1-10.

[3] S. S. Dragomir, N. S. Barnett and P. Cerone, An n-dimentional version of Ostrowski's inequality for mappings of Hölder type, Kyungpook Math. J. 40(2000), 65-75.

[4] M. Matić, J. E. Pečarić and N. Ujević, Weighted version of multivariate Ostrowski type inequalities, Rocky Mountain J.Math. 31(2001), 511-538.

[5] G. V. Milovanović, On some integral inequalities, Univ.Beograd Publ. Elek. Fak. Ser. Mat. Fiz. No.469-No.541 (1975), 119-124.

[6] D. S. Mitrinović, J. E. Pečarić and A. M. Fink, Inequalities for Functions and Their Integrals and Derivatives, Kluwer Academic Publishers, Dordrecht, 1994.

[7] A. M. Ostrowski, Über die Absolutabweichung einer differentiebraen Fuktion von ihrem Integralmitelwert, Comment.Math.Helv. 10(1938), 226-227.

[8] B. G. Pachpatte, On an inequality of Ostrowski type in three independent variables, J. Math. Anal. Appl. 249(2000), 583-591.

[9] B. G. Pachpatte, On a new Ostrowski type inequality in two independent variables, Tamkang J. Math. 32(2001), 45-49.

[10] B. G. Pachpatte, An inequality of Ostrowski type in $n$ independent variables, Facta Univ. (Niš) Ser. Math. Inform. 16(2001), 21-24.

[11] B. G. Pachpatte, On multivariate Ostrowski type inequalities, J. Inequal. Pure and Appl. Math. 3(4)(2002), Art.58.

[12] B. G. Pachpatte, On multidimensional Ostrowski and Grüss type finite difference inequalities, J. Inequal. Pure and Appl. Math. 4(1)(2003), Art.7.

[13] A. Sofo, An integral approximation in three variables, RGMIA Research Report Collection 5(3) (2002), 553-568.

57 Shri Niketan Colony, Near Abhinay Talkies, Aurangabad 431001 (Maharashtra) India.

E-mail: bgpachpatte@gmail.com 